Cahiers « Mondes anciens »

MONDES

ANCIENS

Histoire et anthropologie des mondes anciens

$6 \mid 2015$

Mères grecques

\title{
Mères grecques : des realia à l'imaginaire social et politique des Anciens
}

Ancient Greek Mothers: From Realia to Social and Politic Representations

\section{Florence Gherchanoc}

\section{(2) OpenEdition}

Journals

Édition électronique

URL : http://journals.openedition.org/mondesanciens/1459

DOI : 10.4000/mondesanciens. 1459

ISSN : 2107-0199

Éditeur

UMR 8210 Anthropologie et Histoire des Mondes Antiques

Référence électronique

Florence Gherchanoc, "Mères grecques: des realia à l'imaginaire social et politique des Anciens ", Cahiers « Mondes anciens » [En ligne], 6| 2015, mis en ligne le 28 janvier 2015, consulté le 19 avril 2019 URL : http://journals.openedition.org/mondesanciens/1459; DOI : 10.4000/mondesanciens. 1459

Ce document a été généré automatiquement le 19 avril 2019

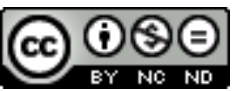

Les Cahiers «Mondes Anciens » sont mis à disposition selon les termes de la licence Creative Commons Attribution - Pas d'Utilisation Commerciale - Pas de Modification 4.0 International. 


\section{Mères grecques : des realia à l'imaginaire social et politique des Anciens}

Ancient Greek Mothers: From Realia to Social and Politic Representations

Florence Gherchanoc

Fig. 1a. Face $A$, amphore attique à figures rouges, vers 470-460.

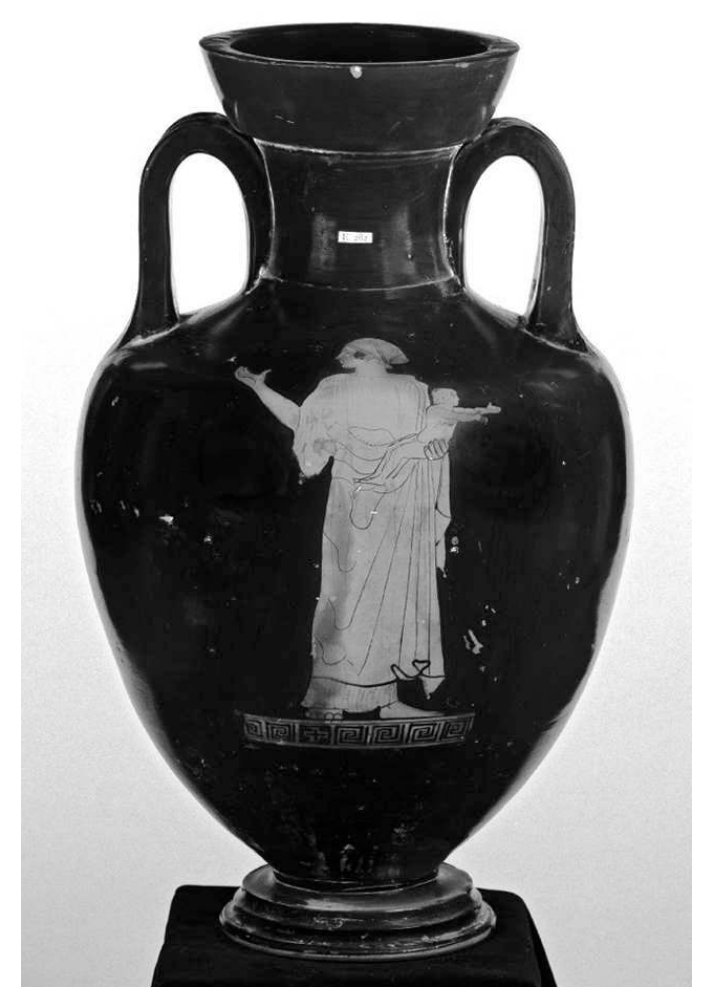

Peintre de Boréas. Londres, British Museum E282. 
Fig. 1b. Face $B$, amphore attique à figures rouges, vers 470-460.

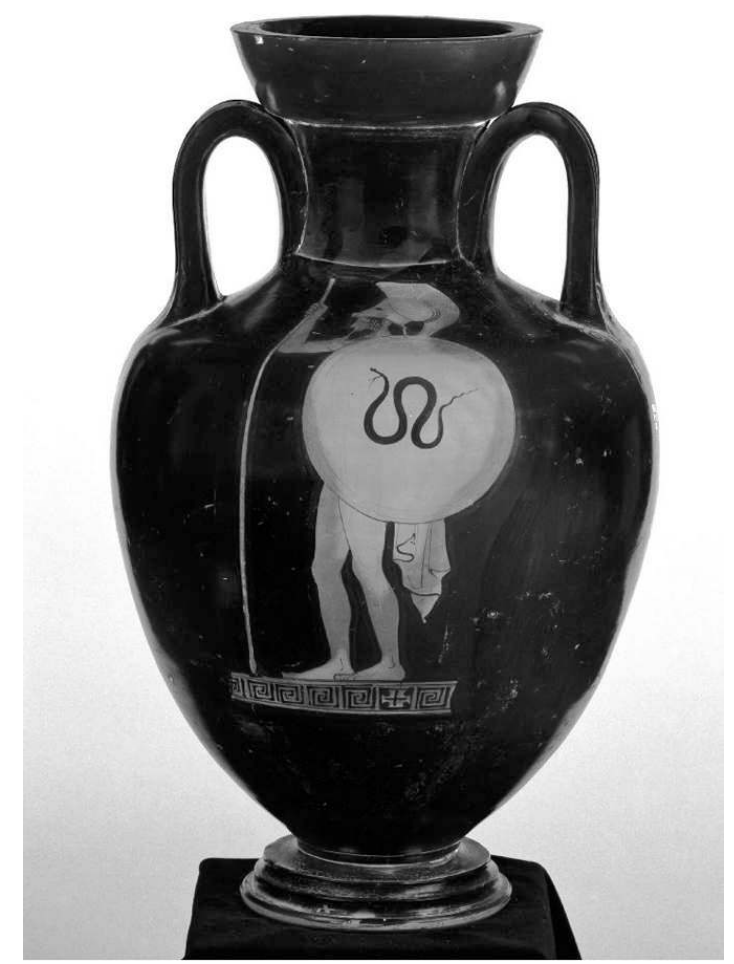

Peintre de Boréas. Londres, British Museum E282.

1 Sur l'une des faces d'une amphore attique à figures rouges attribuée au peintre de Boréas et datée vers 470-460 avant notre ère (Fig. 1a), une femme, vêtue d'un long chiton sur lequel repose un manteau, un sakkos sur la tête, regarde vers la droite et effectue dans cette direction un geste de la main vers son époux; elle soutient de sa main gauche un petit garçon nu qui tend ses deux bras vers la gauche, en direction, de fait, de l'homme barbu, probablement son père, figuré, sur l'autre face du vase, dans un costume d'hoplite (Fig. 1b). Cette scène de départ du guerrier met en exergue une famille "nucléaire", l'épouse et l'époux à chacun desquels est assignée une fonction spécifique comme parent : à l'une, la mère, prendre soin de l'enfant et donc des affaires domestiques, à l'autre, le père, la guerre, pour la protection des siens dont il doit se séparer, des oikoi et de la cité1. Il en émerge, entre autres, une image convenue de la maternité.

2 À peu près à la même époque, Xénophon envisage de manière analogue la répartition des rôles féminin (intérieur) / masculin (extérieur) entre mère et père, deux des éléments constitutifs d'un couple légitimement marié, dont l'association est destinée à faire fructifier l'oikos :

Comme les travaux de la maison aussi bien que ceux du dehors exigent à la fois du labeur et du soin, la divinité, il me semble, a adapté dès l'origine la nature (phusis) de la femme aux travaux et aux soins de l'intérieur, celle de l'homme à ceux du dehors. 23. Froids, chaleurs, marches, expéditions militaires, c'est le corps et l'âme de l'homme qu'elle a constitués de manière à les mieux endurer; aussi lui a-t-elle imposé les travaux du dehors; quant à la femme, la divinité lui a créé un corps moins résistant, aussi elle me semble l'avoir chargée des travaux de la maison. Sachant quelle a accordé au corps de la femme de pouvoir nourrir les nouveau-nés et qu'elle l'en a chargée, elle lui a également donné en partage plus de tendresse 
pour les bébés nouveau-nés qu'elle n'en a donné à l'homme (Xénophon, Économique, VII, 22-24, Paris, Les Belles Lettres, 1949).

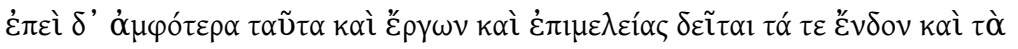

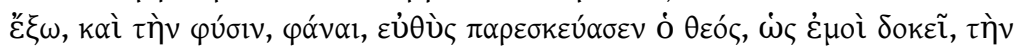

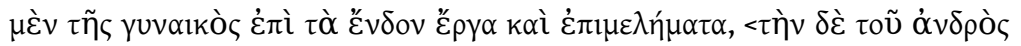

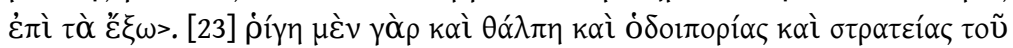

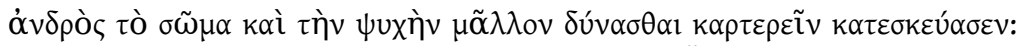

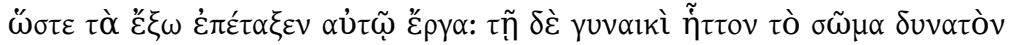

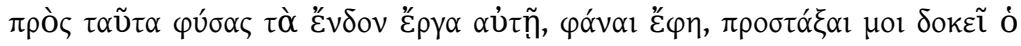

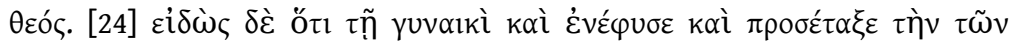

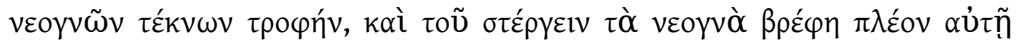

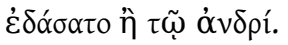

Dans ce registre, il est permis de se demander si l'opinion que ce même auteur développe, également dans les Mémorables, sur la supériorité de l'affection maternelle par rapport à l'amitié est commune ${ }^{2}$ :

Puis l'homme nourrit la femme qui enfantera de lui et il se procure à l'avance, en aussi grandes quantités que ses moyens lui permettent, tout ce qu'il croit devoir être utile à la vie de ses futurs enfants. La femme qui a conçu porte cette charge qui l'alourdit et met sa vie en danger, elle partage avec lui sa propre nourriture et, une fois qu'elle a beaucoup souffert pour le porter à terme et lui donner naissance, elle le nourrit et en prend soin, même s'il elle n'en a reçu auparavant aucun bon traitement, et même si le nourrisson ne sait pas par qui il est bien traité, lui qui n'est même pas capable de signifier ce dont il a besoin ; mais elle, devinant ce qui lui est utile et agréable, s'efforce de combler ses besoins ; elle le nourrit longtemps en endurant de jour et de nuit la fatigue, et sans savoir si cela lui vaudra en retour un peu de reconnaissance (Xénophon, Les Mémorables, II, 2, 5, Paris, Les Belles Lettres, 2011).

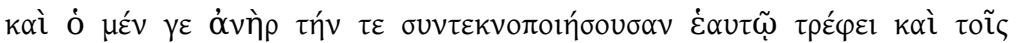

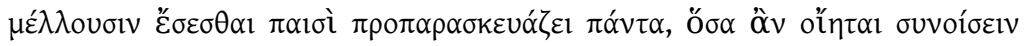

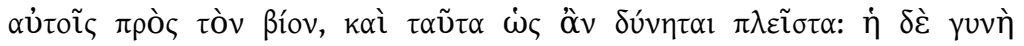

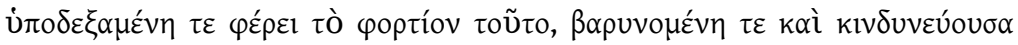

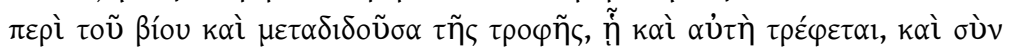

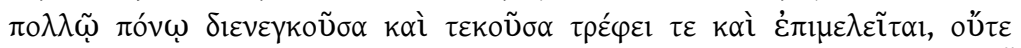

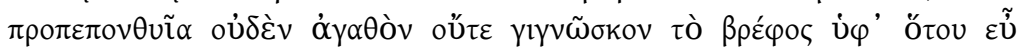

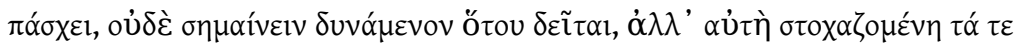

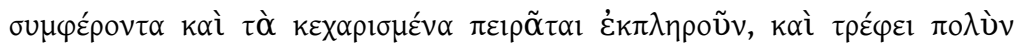

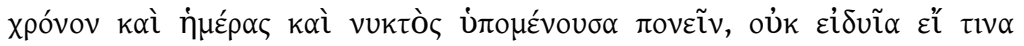

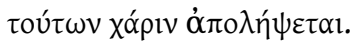

4 La maternité (capacité reproductrice et nourricière des femmes, ainsi qu'aptitude aux soins des petits) serait un fait de nature et, en ce sens, qualifie une propriété biologique et naturelle propre aux individus de sexe féminin. Cette image stéréotypée de la maternité construite par Xénophon durant la première partie du IV siècle av. J.-C., fondée sur des critères de genre, est-elle universelle, si l'on considère l'Athènes classique, puis plus largement le monde grec antique?

En d'autres termes, qu'est-ce qu'une mère? Une femme qui fait croître en elle un embryon, porte un bébé, l'engendre et le nourrit; une femme qui, en raison d'une tendresse naturelle, prodigue des soins maternels et protège ses enfants; une femme, pourrait-on ajouter, qui pleure la mort de sa progéniture plus que tout autre comme le 
suggèreraient, par exemple, les lois sur le deuil (cf. Loraux 1993 ; Gherchanoc 2011) et qui parfois les venge (Schmitt Pantel 2013)?

6 Cette description relativement simpliste, que proposent encore, en partie, nos dictionnaires des $\mathrm{xx}^{\mathrm{e}}$ et $\mathrm{xxI}^{\mathrm{e}}$ siècle ${ }^{3}$, est loin d'être satisfaisante. Le monde grec des cités connaît, par exemple, toute une série de contre-modèles relatifs aux realia comme à l'imaginaire ${ }^{4}$. Il n'est pas question de nier, bien sûr, que la maternité est notamment une réalité biologique mais de montrer que celle-ci est aussi une construction sociale. Les textes et les images, suivant des logiques génériques et narratives diverses jouent sur ces polarités, à différentes échelles, pour proposer des tableaux contrastés des mères et des maternités. Ces aspects, précisément, nous ont particulièrement intéressé et explicitent les raisons pour lesquelles une approche historique et anthropologique a été privilégiée pour analyser les mères et les formes de maternité. Aussi, dans ce numéro des Cahiers "Mondes Anciens» qui réunit les actes de deux journées d'étude internationales ${ }^{5}$, est-il question des mères et des maternités au pluriel car ces femmes ou figures maternelles, humaines comme divines, sont envisagées dans la pluralité des constructions culturelles qui les concernent et les mettent en scène, en un temps donné et dans une société grecque spécifique. Enfin, analyser les mères grecques sous ce prisme a conduit à discuter pour les réévaluer une certaine survalorisation de la paternité et, ainsi, la place occupée par les mères et la maternité, au sein de sociétés dites patriarcales, dans les pratiques effectives - sociales, religieuses et politiques - et dans l'imaginaire des anciens Grecs. À côté des discours où la femme, ce «mal nécessaire " pour perpétuer l'espèce, voit, le temps d'une manipulation discursive, son rôle minimisé voire occulté au profit de la construction d'un monde où les hommes et les pères ont la plus belle part, voire l'exclusivité, n'en existe-t-il pas d'autres qui suggèreraient que les mères - sous contrôle masculin, sans doute dans bien des cas - disposaient d'une certaine latitude d'action et de formes associées de reconnaissance sociale, religieuse et politique en raison de leur statut ? De la pratique à l'imaginaire social et politique, l'articulation, le lien, la proximité comme l'écart, méritent dès lors d'être interrogés. Nous avons privilégié, pour ce faire, des thèmes qui nous ont semblé peu ou pas étudiés jusqu'à présent.

7 Le dossier explore suivant ces perspectives, plus spécifiquement, mais pas uniquement, pour les époques archaïque et classique, trois dimensions des maternités grecques. La première a trait à la relation mère-enfant (fille et garçon, humain et dieu) sous l'angle de la trophê, de la courotrophie, de la protection et de la transmission de modèles comportementaux comme de la fabrication de l'identité. La deuxième concerne la visibilité religieuse (en particulier sur l'Acropole d'Athènes), juridique (à Gortyne et Athènes) et politique (chez Hérodote et Plutarque) des femmes, dans l'espace public, dans leur fonction de mères. La troisième porte principalement sur la question des maternités d'Athéna, figure, a priori, improbable de mère en raison de sa qualité de divinité parthenos ; et pourtant... 


\section{BIBLIOGRAPHIE}

Bonnard J.-B. (2004), Le complexe de Zeus. Représentations de la paternité en Grèce ancienne, Paris.

Bonnard J.-B. et Gherchanoc F. éd. (2013), Mères et maternités en Grèce ancienne, Mètis N. S. 11, p. 5-144.

Chazalon L. et Wilgaux J. (2008-2009), «Violences et transgressions dans le mythe de Térée », AION n. s. 15-16, p. 167-189.

Cid López R. M. éd. (2009), Madres y maternidades. Construcciones culturales en la civilización clássica, Oviedo.

- éd. (2010), Maternidad/es : representaciones y realidad social. Edades antigua y media, Madrid.

Damet A. (2012), « La part du féminin et du masculin dans l'infanticide : des realia aux représentations tragiques (Athènes, époque classique) », dans Dubel S. et Montandon A. éd., Mythes sacrificiels et ragoûts d'enfants, Clermont-Ferrand, p. 315-327.

Demand N. (1994), Birth, Death, and Motherhood in Classical Greece, Baltimore-Londres.

Dermenjian G., Guilhaumou J. et Lapied M. éd. (2008), La puissance maternelle en Méditerranée. Mythes et représentations, Arles.

Difabio de Raimondo E. H. (2000), « Existencia mítica de personajes infanticidas : los cucos [cucas] griegos », Circe V, 2000, p. 101-116.

Gherchanoc F. (2011), « Mise en scène et réglementations du deuil en Grèce ancienne », dans Sebillotte Cuchet V. et Ernoult N. éd., Les femmes, le féminin et le politique après Nicole Loraux, Classics@ [en ligne] issue 7, 2011. URL : http://chs.harvard.edu/wa/pageR? tn=ArticleWrapper\&bdc=12\&mn=3369

Gherchanoc F. et Bonnard J.-B. (2013), « Mères et maternités en Grèce ancienne. Quelques éléments historiographiques et pistes de réflexion », dans Bonnard et Gherchanoc éd. 2013, p. 7-28.

Halm-Tisserant M. (1994), « Festin cannibale. La mère égorgeuse », Kentron X/1, 1994, p. 59-70.

Klöckner A. (2005), « Mordende Mütter : Medea, Prokne und das Motiv der furchtbaren Rache im klassischen Athen ", dans Fischer G. et Moraw S. éd., Die andere Seite der Klassik. Gewalt im 5. und 4. Jahrhundert v. Chr, Stuttgart, p. 247-263.

Landucci Gattinoni F. (2008), « Agatocle, Ofella e il mito di Lamia : (Diod. 20.41.2-6) », Aristonothos II, 2008, p. 161-175.

Lissarrague F. (1990), L'autre guerrier. Archers, peltastes, cavaliers dans l'imagerie attique, Paris-Rome.

Loraux N. (1989), « Le lit, la guerre », dans Les Expériences de Tirésias. Le féminin et l'homme grec, Paris, p. 29-53.

- (1990), Les mères en deuil, Paris.

Lütkehaus L. (2009), « Der Medea-Komplex : Mutterliebe und Kindermord », dans Zimmermann B. éd., Mythische Wiederkehr : der Ödipus- und Medea-Mythos im Wandel der Zeiten, Fribourg

(Allemagne)-Vienne, p. 121-133. 
Peek W. (1955), Griechische Vers-Inschriften, Berlin.

Pérez Miranda I. (2010), « Madres terribles : avaricia, envidia, traición y mentira en la mitología griega », dans Cid López éd. 2010, p. 59-74.

Peterson L. H. et Salzman-Mitchell P. éd. (2012), Mothering and Motherhood in Greece and Rome, Austin.

Schmitt Pantel P. (2013), « L'histoire de Damocrita dans les Histoires d'amour de Plutarque : la vengeance d'une mère épouse de citoyen à Sparte ", dans Boehringer S. et Sebillotte Cuchet V. éd., Des femmes en action, Mètis H. S. 1, p. 185-198.

Thébaud F. et Knibiehler Y. éd. (2005), Maternités, Clio, HFS 21.

Walcot P. (1987), « Plato's Mother and Other Terrible Women », G\&R 34, 1987, p. 12-31.

\section{NOTES}

1. Sur la femme et le guerrier, voir Lissarrague 1990, p. 43-47. Sur la dichotomie des destins des femmes et des hommes, voir Loraux 1989.

2. Sur le caractère inconditionnel et non réciproque de l'amour des parents pour leurs enfants, cf. Platon, Lysis, 213a (Paris, Les Belles Lettres, 2004) ; Aristote, Éthique à Nicomaque, VIII, 9, 1159 a 27-33 (Garnier-Flammarion, Paris, 1965).

3. Ce modèle dit traditionnel a été produit au XIX ${ }^{\mathrm{e}}$ siècle par une société bourgeoise; il ne correspond pas toujours, pour ne pas dire de moins en moins, aux usages propres aux sociétés occidentales contemporaines (cf. la monoparentalité; les familles recomposées; l'homoparentalité ; les mères porteuses ou gestatrices ; la parenté biologique ; la parenté légale, etc.) ni, dans certains cas, à la loi qui s'adapte progressivement aux transformations sociales. Ni $a$ fortiori à ceux des cités grecques de l'Antiquité.

4. Voir, par exemple Bonnard 2004, sur le rêve d'une paternité quasi exclusive avec à l'extrême des figures de pères "parturients » et les représentations d'une maternité à la fois passive et dépréciée dans les discours mythiques et les discours scientifiques. Pensons, en outre, aux ogresses, aux infanticides, aux incestueuses - Lamia, Médée, Procné et les autres - qui comme mères exemplaires sont peut-être loin d'incarner de «bonnes » mères; voir, en particulier, sur les ogresses: Halm-Tisserant 1994; Landucci Gattinoni 2008; sur les infanticides: Difabio de Raimondo 2000 ; Klöckner 2005 ; Chazalon et Wilgaux 2008-2009; Lütkehaus 2009 ; Damet 2012 ; sur les incestueuses : Walcot 1987 ; Pérez Miranda 2010. Pensons, enfin, à ces pères qui pleurent aussi leurs enfants, comme en témoignent des épigrammes funéraires : cf. Peek 1955, n 1989, à propos de Téiophilè fille d'Hektaios.

5. Ces rencontres scientifiques se sont déroulées le 6 octobre 2012 et le 16 novembre 2013 à l'INHA. Elles s'inscrivaient dans un programme de recherche initié en 2010-2011, mené en collaboration avec Jean- Baptiste Bonnard et financé en grande partie par la dotation de l'IUF de F. Gherchanoc. Les actes de la première journée d'étude du 10 mars 2012, consacrée principalement au fait de « Devenir mère » autour des questions du contrôle des naissances, des liens entre maternité et santé, du rôle des divinités dans le processus d'engendrement, du cas d'une maternité précoce, souhaitée ou avortée, ont été publiés dans la revue Mètis avec un bilan historiographique et une présentation de la problématique générale qui ne sont pas, pour cette raison, repris dans ce volume : voir Bonnard et Gherchanoc 2013 ; Gherchanoc et Bonnard 2013. Rappelons juste pour mémoire et comme jalons les travaux suivants : Demand 1994 ; Thébaud et Knibiehler 2005 ; Dermenjian, Guilhaumou et Lapied 2008 ; Cid López 2009 et 2010 ; Peterson et Salzman-Mitchell 2012. 
INDEX

Mots-clés : mère, Grèce ancienne, maternité, Athènes, mariage, fête de famille

Keywords : maternity, mother, motherhood, Greek Antiquity, Athens, marriage, family festival

\section{AUTEUR}

\section{FLORENCE GHERCHANOC}

Université Paris Diderot-Paris 7, ANHIMA - UMR8210 